\title{
Agôn
}

Revue des arts de la scène

1 | 2008

Interstices, entractes et transitions

\section{Rythmes, air et tissus blancs : l'art des transitions entre les cultures dans Bamboo Blues de Pina Bausch}

\section{Katia Légeret}

\section{(2) OpenEdition}

Journals

Édition électronique

URL : http://journals.openedition.org/agon/719

DOI : 10.4000/agon.719

ISSN : 1961-8581

Éditeur

Association Agôn

Référence électronique

Katia Légeret, "Rythmes, air et tissus blancs : l'art des transitions entre les cultures dans Bamboo Blues de Pina Bausch », Agôn [En ligne], 1 | 2008, mis en ligne le 18 décembre 2008, consulté le 02 juin 2020. URL : http://journals.openedition.org/agon/719; DOI : https://doi.org/10.4000/agon.719 


\section{Rythmes, air et tissus blancs : l'art des transitions entre les cultures dans Bamboo Blues de Pina Bausch}

Bamboo Blues ${ }^{l}$, la dernière création de Pina Bausch présentée à Paris en 2008 au Théâtre de la Ville, pousse à l'extrême l'un des principes du Tanztheater - une succession rapide et non narrative de plusieurs dizaines de saynètes - en faisant de la transition une idée essentielle de sa mise en scène. Entre l'Inde ${ }^{2}$ et l'Europe, elle interroge en effet d'une manière originale l'interculturalité, inventant selon l'expression deleuzienne, de nouveaux « passages d'un milieu dans un autre ${ }^{3} »$.

Ces transitions artistiques et esthétiques sont fabriquées à trois niveaux : temporel, par des collages d'intervalles rythmiques dans et entre les scènes, visant à fracturer par leurs vitesses les clichés culturels du spectateur ; spatial, avec des tissus sans cesse pliés et dépliés, jouant dans leurs interstices à détourner les objets de leurs fonctions (rideau, écran, jupe, drap, châle, serviette...) par des agencements de fragments de vie puisés dans le quotidien ; relationnel, avec l'élément air, transformant les frontières instituées (interdits, tabous, formes, corps) en échanges de blocs de sensations et d'affects.

Cet art de la transition témoigne d'une posture originale dans la mise en scène contemporaine. Entre la danse, le théâtre et les projections du vidéaste Peter Pabst, Pina Bausch joue sur la présence infiniment réitérée de l'instant, dont la pulsation se nomme, entre les cultures, apparition/disparition, formation/déformation, agencement/dissolution, actualité/ virtualité, détournant ainsi le spectateur de toute forme de fascination passive et a priori. Ce procédé fonctionne différemment d'une représentation à l'autre puisqu'au cours des tournées de Bamboo Blues, l'ordre des scènes a été changé ainsi que les combinatoires entre les images projetées, les actions des danseurs et les couleurs des costumes.

Réfléchir sur les intervalles entre les scènes de Bamboo Blues implique un questionnement préalable sur la fonction du rythme dans cette création. L'origine grecque du mot, rhutmos, en donne deux sens distincts ${ }^{4}$ : le rythme est à la fois une manière particulière

\footnotetext{
${ }^{1}$ Après sa création en Inde suivie d' une tournée dans ce pays du 4 au 19 janvier 2008, Bamboo Blues a été joué au Théâtre de la Ville de Paris du 16 juin au 2 juillet 2008. Puisque des variations de mise en scène existent d'un soir à l'autre, nous précisons que notre article se fonde sur la représentation du 2 juillet.

${ }^{2}$ Pina Bausch et le Tanztheater en tournée, film de Anne Linsel, Arte/De campo film, Köln, 1994 : ce film relate leur voyage en Inde de 1994 pour jouer Nelken. Pina Bausch y découvre « de formidables richesses, avec une telle qualité de formes, de détails, dont nous avons tous quelque chose à apprendre. (...) J'ai parlé avec beaucoup de gens qui m'ont semblé être dotés de forces inépuisables, alors que chez nous, les gens sont tristes et épuisés (...) D'où cela vient-il ? Quelles sont nos erreurs ? Comment et pourquoi est-ce autrement ici ? (...) J'aimerais tant apprendre tout ce que j'ai ressenti ici (...) Ce désir et ce bonheur de savoir que tout cela existe, c'est déjà magnifique. Le fait d'aspirer à tout cela est aussi très important ». Précisons que Pina Bausch s'exprime dans ce film en anglais mais le sous-titrage est en français.

${ }^{3}$ Gilles Deleuze et Félix Guattari, Mille Plateaux, coll. «Critique », éd. De Minuit, Paris, 1980, p. 385.

${ }^{4}$ Sur cette double signification du mot rythme, $C f$. l'ouvrage collectif Rythmes et philosophie, sous la direction
} 
de s'écouler et la mesure de ce qui revient régulièrement. Dans le premier cas, les formes momentanées du flux temporel correspondent à la multitude des petites scènes puisées dans le réel ou dans le rêve et s'enchaînant dans Bamboo Blues : elles donnent un sentiment de continuité tant leur vitesse de succession est grande. La question sera de savoir comment procède ce collage de séquences pour comprendre un tel paradoxe.

Face à cette variation ininterrompue de saynètes au contenu sans cesse différent, le second sens du mot rythme suggère un ordre imposé à ce mouvement : le retour périodique d'une forme précise, celle d'un intervalle entre chaque scène. Elément le plus récurrent du spectacle et le plus égal à lui-même, il contrebalance la sensation qu'a le spectateur d'être emporté dans un enchaînement de situations sans logique narrative. Par sa double nature spatiale et temporelle, cet intervalle se définit à la fois comme une césure rythmique extrêmement rapide et comme interstice spatial : sans cesse, certains danseurs apparaissent au moment où d'autres disparaissent dans le noir, à tous les niveaux des coulisses, sur les côtés de la scène et dans les rideaux du fond. La scène ne se vide qu'à l'entracte, laissant le temps aux techniciens de recouvrir le sol noir par un tapis de danse blanc. A ces deux lectures apparemment contradictoires du rythme correspondent deux artifices dramaturgiques, fabriquant des interstices pratiquement invisibles pour les spectateurs. Ils feront l'objet de deux analyses successives. Le premier artifice se fonde sur les courses circulaires des danseurs et la seconde sur les modes de circulation transversale induits par l'élément air.

\section{Les interstices créés par les mouvements circulaires : l'illusion d'une conti- nuité}

Cette alternance si rapide de passages est accomplie par les danseurs entre le noir des coulisses et les feux de la scène. Celle-ci reste toujours éclairée, le rythme s'inscrit en tant que moment critique entre le continu et le discontinu. ${ }^{5}$ Tout le déroulement de Bamboo Blues s'accomplit sur des enchaînements de musiques d'inspiration indienne très rythmées et variées dans leurs vitesses. Or, les transitions entre les morceaux ne correspondent pas toujours exactement aux passages en coulisse des danseurs. Mais la sensation intense et permanente des rythmes musicaux est redoublée par ces entrées et sorties qui se comptent par dizaines soixante-dix scènes incluant en plus une trentaine d'apparitions/disparitions des artistes. Cette pratique du rythme comme "coupure » répond à la nécessité de briser toute référence aux clichés sur l'Inde, aux jugements de valeur et aux métaphores. Le procédé de Pina Bausch est simple : choisir une image toute faite sur l'Inde, l'image captive d'un comportement rituel, d'une gestuelle codée, le souvenir d'une scène quotidienne de rue et la miner de l'intérieur par des ruptures de rythme, tels des contretemps. Pina Bausch met notamment en pratique ces « coupures » dans certaines scènes fondées sur la répétition de mouvements circulaires. Les quatre éléments dramaturgiques que nous analysons ci-après développent ce procédé : les trajets circulaires rapides, répétitifs et accomplis par les mêmes danseurs ; les trajets circulaires enchaînant des actions sans lien narratif ; les petits cercles de lumière sur le plateau

de Pierre Sauvanet et de Jean-Jacques Wunenburger, éd. Kimé, Paris, 1996.

${ }^{5}$ Pierre Boulez donne une définition éclairante de ce moment critique : « le continuum se manifeste par la possibilité de couper l'espace suivant certaines lois ; la dialectique entre le continu et le discontinu passe donc par la notion de coupure. J'irais jusqu'à dire que le continuum est cette possibilité même », in Pierre Boulez, Penser la musique aujourd'hui, éd. Gontier, Paris, 1963, p. 95. Bamboo Blues enchaîne une grande variété de morceaux musicaux indiens édités très récemment avec des compositeurs, formés depuis l'enfance à un style traditionnel, tels Anoushka Shankar au sitar par son père Ravi Shankar, Sunil Ganguly à la musique hindoustanie puis à la guitare électrique hawaïenne, Talvin Singh aux tablas. 
isolant des actions différentes ; les cercles liés à la culture indienne et déconstruits par le rythme.

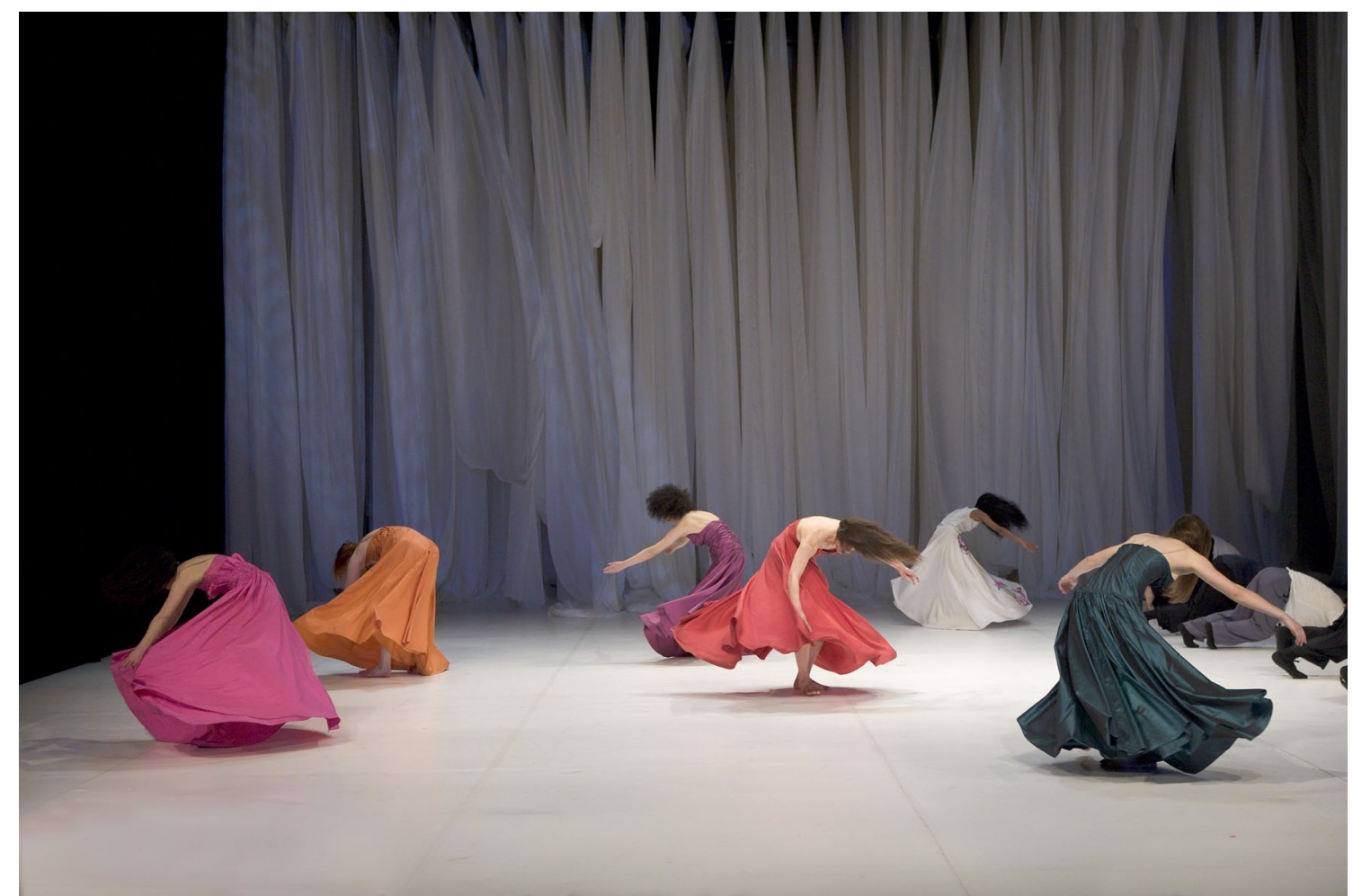

Bamboo Blues, Pina Bausch - (C) Théâtre de la Ville, Paris

\section{Evolutions circulaires et répétitives}

Les séquences enchaînant des cercles sont agencées comme des passages. Leur répétition dans une même scène, au même rythme et par les mêmes danseurs est très captivante. Mais l'entrée inattendue d'un ou plusieurs danseurs commençant une action particulière détourne brusquement l'attention du spectateur. Il ne voit pas que les trajectoires circulaires se sont un peu élargies au point de se prolonger au bord des rideaux puis vers les coulisses. Lorsque les coureurs réapparaissent quelques scènes plus loin, il a l'impression que ces circonvolutions n'ont pas cessé de se faire dans les coulisses ou ailleurs. Il s'agit à chaque fois d'un couple qui effectue un ou plusieurs cercles sur scène d'un pas rapide ou en courant. Ces couples reviennent régulièrement, cinq fois dans la première partie et cinq dans la seconde. Deux de ces scènes se répètent presque à l'identique : l'une montre deux hommes enlacés et l'autre une femme entraînée par un homme à sauter sur une chaise.

Les gestuelles des danseurs sont puisées dans la vie quotidienne indienne mais elles ne peuvent plus être perçues comme telles par le spectateur. En effet, des ruptures rythmiques s'y inscrivent. Elles proviennent de la très grande vitesse de déplacement des danseurs, incompatible avec l'action de leurs mains et l'expression de leur visage. Par exemple dans la première scène, les deux hommes s'enlacent tendrement, tout en se mettant à courir côte à côte en décrivant de grands cercles. Leurs pas deviennent de plus en plus mécaniques, parfaitement réglés entre eux, les genoux anormalement remontés vers l'avant, leur buste se fige dans la même position et leurs visages restent impassibles. Ces actions scéniques 
tranchent avec toutes celles qui présentent des femmes aux marches mesurées, en pure représentation, défilant en longues robes de soirées, multipliant des sourires séducteurs adressés au public ou aux danseurs. Dans le cas des couples rapides évoluant en cercles, il n'y a plus d'adresse aux spectateurs. Ceux-ci sont simplement témoins d'un passage, sans connaître l'origine ni la destination de ces êtres humains entraînés dans des circonvolutions dont on ne connaît ni l'origine ni la destination.

\section{Collages de cercles sans liens narratifs}

Dix mouvements circulaires, correspondant à dix scènes différentes, se présentent successivement de la manière suivante. Un homme entre en courant, il porte sur son dos, à la manière d'un sac, une femme recroquevillée sur elle-même. Le fait de courir avec ce « fardeau », dont les secousses introduisent des contretemps curieux, rend la scène inquiétante et incompréhensible. Il est possible que le spectateur ne voie pas entrer cet étrange binôme, car du côté opposé une scène s'achève avec deux femmes quittant lentement le plateau. Plus loin dans le spectacle, un homme fait le tour de la scène en patins à roulettes. Comme il est torse nu, les hanches moulées dans une sorte de jupe longue - un vêtement traditionnel appelé dhoti -, cette apparence rend le jeu à la fois périlleux et drôle. Il apparaît dans une autre scène où Shantala Shivalingappa, connue en tant qu'artiste de Kuchipudi - style de théâtre dansé traditionnel de l'Inde du sud - entre en robe longue avec une démarche et des gestes de mains inspirés par les codes de cet art indien (les $m u d r a ̂$ ). Elle se déplace au ralenti, les bras et les cuisses entourés de guirlandes électriques, les petites ampoules jaunes allumées : l'homme en patins à roulettes se met à pivoter autour d'elle. Tradition et modernité se côtoient ainsi dans leurs accessoires de façon ludique.

Un homme entre en dansant, enlacé par une femme en robe longue. Un second danseur surgit, tire de force la femme et l'entraîne dans une course folle, l'obligeant à sauter sur une chaise qui se trouve sur ce trajet circulaire. Entraînée par son élan, elle tombe à chaque fois dans les bras du danseur. Ce qui pourrait être un simple jeu rythmé par l'élan du saut et le temps fort de leur embrassement, est miné par l'effort, la fatigue, la peur et la douleur qui se lisent sur le visage de cette danseuse. Dans un dernier élan désordonné, elle tombe vers les coulisses. Cette rupture rythmique violente dans cette chute libre des corps n'est pas vécue comme telle par le spectateur car avant leur disparition, la percussion régulière et harmonieuse des tablas indiens annonce le solo d'un danseur qui surgit alors. Plus loin encore, deux hommes portent sur leur épaule la danseuse indienne Shantala, hiératique et inexpressive. Une image surgit spontanément : celle d'une déesse sortie d'un temple, le temps d'une procession autour du lieu sacré ou du village. Mais la rapidité des coureurs rend caduque la relation au cérémonial hindou régi par la lenteur et la mesure.

Dans une autre scène, alors qu'elle vient de s'allonger doucement sur le plateau, un homme tient cette même danseuse indienne par les pieds et il tourne à toute vitesse sur luimême, l'entraînant dans sa giration et la soulevant de terre. Tenant une craie dans la main, elle dessine au gré de ses voltes des grands cercles blancs sur le plateau. Un signe de sa liberté de mouvement renversant les formes préétablies ? Ou à l'inverse, un corps-compas soumis aux déplacements d'un homme et incapable de se tenir debout ? La majorité de ces scènes construites sur un principe circulaire sont précédées et suivies de séquences très narratives, comprenant un grand nombre d'événements et de danseurs. Par conséquent, ces actions circulaires rapides avec un ou deux danseurs, créent une sorte d'interstice : il s'agit d'un espace 
vide, visible pour le spectateur au centre des cercles accomplis par les danseurs, marquant une pause entre des bribes d'histoires ou une fracture rythmique interrompant brutalement la ligne d'un récit possible.

\section{Jeu de cercles lumineux}

L'omniprésence des structures circulaires est redoublée par le rythme rapide de petites scènes éphémères mises en valeur par une intensité lumineuse particulière, le protagoniste étant placé sous une " douche » de lumière. Elles se comptent par dizaines dans ce spectacle, agençant par collage des fragments de scènes quotidiennes. Entourer de lumière chacun de ces morceaux, avec des cercles lumineux de tailles différentes, permet au spectateur une certaine sérénité dans cet univers poétique : il est en effet invité à voyager dans une culture étrangère, à y circuler, tout en restant à la fois radicalement différent et à l'extérieur. Mais ce procédé joue également avec l'idée de frontière isolante, vue comme une limitation ou un interdit entre deux sphères antagonistes. L'un des fondamentaux du théâtre indien selon le Nâtyashâstra ${ }^{6}$ concerne les usages du mouvement circulaire des acteurs-danseurs sur scène, principalement réservés aux changements de rôles, de mondes, aux combats martiaux et aux cercles magiques de protection ${ }^{7}$. Bamboo Blues suggère au spectateur une signification ambivalente de cette notion de limite tracée par le cercle. Certes, les « douches » de lumière isolent à chaque fois une action particulière. Mais comme elles sont rapides et s'allument ici et là sur le plateau, le spectateur est autant captivé par leur surgissement que par les circulations entre ces cercles lumineux. En effet, les danseurs continuent leur mouvement dans l'ombre ou se dirigent vers les coulisses et les spectateurs scrutent ces intervalles obscurs où quelque chose se passe, un geste s'esquisse tout en restant incompréhensible de l'extérieur.

\section{Briser les clichés culturels}

Les deux sens grecs du mot ruthmos définis en introduction permettent de dissocier ces deux modes de composition : la répétition de formes fondées sur la symétrie ou l'addition, et la prolifération infinie et indéfinie de formes fluides et éphémères. Dans Bamboo Blues, le cercle dansé est récurrent une douzaine de fois. Sa mise en scène rappelle à un premier niveau de lecture l'importance des pratiques indiennes basées sur des mouvements circulaires, tant dans l'art que dans la vie quotidienne. Par exemple, les kolams, ces dessins de poudres colorées tracés chaque matin par les femmes indiennes devant leur maison. Mais en rester là réduirait l'interculturalité à la projection d'un espace pré-ordonné par une mesure extérieure et conventionnelle. Cet espace "strié » selon la terminologie deleuzienne ${ }^{8}$, sous-tend les trajets des danseurs sur la scène d'une grille symbolique et culturelle obéissant à une métrique qui clôture et stabilise le lieu.

\footnotetext{
${ }^{6}$ Nâtyashâstra, trad. angl. et éd. M. Ghosh, Royal Asiatic Society of Bengal, Calcutta, 1951 (Vol. I) et 1967 (Vol. II).

${ }^{7}$ Katia Légeret, Esthétique de la danse sacrée, éd. Geuthner, Paris, 2001, pp. 60-70.

${ }^{8}$ Gilles Deleuze et Félix Guattari, Qu'est-ce que la philosophie, coll. « Critique », éd. de Minuit, Paris, 1991, p. 172 : ces auteurs empruntent la distinction espace lisse/espace strié à Pierre Boulez (Penser la musique, op. cit., note 5) et l'association entre l'espace lise et la ligne nomade à Wilhelm Worringer (Art gothique, 1927, trad. fr. D. Decourdemanche, éd. Gallimard, Paris, 1941, rééd. Coll. « idées/art », 1967). Ces références sont analysées par Anne Sauvagnargues dans son ouvrage Deleuze et l'art, éd. PUF, coll. « Lignes d'art », Paris, 2005, pp. 228-240.
} 
Par opposition, l'espace « lisse » déploie des lignes nomades, des torsions dynamiques, des spirales qui accélèrent les transformations. Dans ce contexte, les transitions de Bamboo Blues ne seraient pas réductibles au passage d'une scène à l'autre, présupposant des formes préétablies. Mais elles témoigneraient du "passage d'un milieu dans un autre ${ }^{9}$ », par l'effacement des sujets et des objets et l'émergence d'une scène de vie en tant que capture de forces et variations d'affects.

La triple action du danseur - entrer / faire un cercle / sortir - ne consiste pas en une translation de corps d'ici à là-bas, ni en un objet stable circulant dans un espace global. Elle devient vibratoire, ces trois temps étant rythmés par des transformations intensives, des qualités sensitives non mesurables, des sous-rythmes venant le miner et le fracturer dans sa répétition circulaire. Gilles Deleuze nomme ce processus un " devenir-ligne », présent d'après ses analyses dans la peinture chinoise : reposant non pas « sur des points ou des objets, mais sur des heccéités, sur des ensembles de relations (vents, ondulations de la neige et du sable) ${ }^{10}$, le paysage entre montagne et eau montre que « la lumière des fumées et l'encre des nuées sont sans cesse changeantes ${ }^{11} \gg$.

Si Pina Bausch sait comment briser le cliché par des ruptures de rythme, la création d'un espace lisse existe également dans le théâtre dansé indien, à la manière d'une porte de sortie vers la puissance des forces, loin de l'assujettissement des formes ${ }^{12}$. L'interculturalité joue à ce niveau dans l'action de discréditer les images toutes faites ${ }^{13}$. Elle interroge également les styles artistiques traditionnels sclérosant les danseurs par leurs codes. Shantala Shivalingappa dément sur scène une telle pensée puisqu'elle est à la fois danseuse contemporaine et interprète de kuchipudi traditionnel ${ }^{14}$. Dans la scène de Bamboo Blues où ses membres sont ornés de guirlandes électriques, elle exécute un ensemble de gestes codés signifiant dans le kuchipudi une étendue d'eau : yeux en forme de lotus, bouche qui évoque le bouton de fleur qu'une abeille vient butiner. Mais son langage gestuel n'est plus narratif : les yeux souvent fermés, elle laisse faire une mémoire corporelle qui déplace les mains sur un mode aléatoire, avec des répétitions accélérées, des changements de directions inattendus. Les glissements de sens qui en résultent fracturent une histoire qui ne serait plus à lire. La liberté dont témoigne cette danseuse pour composer sa gestuelle loin de toute référence littéraire présuppose néanmoins une grande maîtrise préalable de l'improvisation en kuchipudi qui, elle, se fonde sur l'interprétation d'un texte. Avec cette artiste, Bamboo Blues met en scène la question d'une interculturalité fabriquée par les transitions entre certains gestes codés et la transformation de leurs significations, tout en préservant la grâce et la précision de cet art traditionnel.

\footnotetext{
${ }^{9}$ Gilles Deleuze et Félix Guattari, Mille Plateaux, op. cit., p. 385.

${ }^{10}$ Ibid., pp. 321 et 474. Le mot heccéité est définit ainsi par Gilles Deleuze dans Dialogues, éd. Flammarion, 1977, p. 111 : « toute individuation (qui) ne se fait pas sur le mode d'un sujet ou même d'une chose ».

${ }^{11}$ François Cheng, Vide et plein, le langage pictural chinois, éd. du Seuil, Paris, 1979, rééd. Coll. « Points », 1991, p. 91.

${ }^{12}$ Dans un répertoire traditionnel, les morceaux de danse pure (nritta), non liés à la narration d'un texte, expérimentent cet espace lisse : $C f$. Katia Légeret, La gestuelle des mains dans le théâtre dansé indien, éd. Geuthner, Paris, 2004, pp. 104-112.

${ }^{13}$ Bamboo Blues se joue ainsi beaucoup des clichés culturels en détournant les gestes de leur fonction initiale : par exemple, des jeunes filles s'emparent des longues écharpes des garçons pour les transformer en cravates.

${ }^{14}$ Le kuchipudi est un style de danse originaire de l'Inde du Sud (Andhra Pradesh). Shantala Shivalingappa est aussi comédienne, et apparaît notamment dans les mises en scène de Peter Brook.
} 


\section{Les passages de l'élément Air : un jeu de signes}

Les créations de Pina Bausch commencent souvent par ce même choix scénographique où un élément de la nature est mis en valeur : une nappe d'eau (Arien, 1979), un gazon (1980, 1980), un champ d'œillets (Nelken, 1982), un désert parsemé de cactus géants (Ahnen, 1987), de la neige qui tombe (Tanzabend II, 1991), ou encore une végétation tropicale (Agua, 2001). Dans Bamboo Blues, l'élément représentant la nature est quasiment invisible et seulement sensible dans les mouvements qu'il provoque chez les choses et les êtres qui l'entourent. Il s'agit tout simplement de l'air. La modernité assure de nouveaux moyens de résistance au climat extrême de l'Inde, comme en témoigne une scène de ce spectacle où des badauds s'attroupent autour d'étagères roulantes remplies de grands ventilateurs électriques. Ce progrès n'intéresse pas seulement les humains mais aussi les dieux et les animaux. En effet, un homme avec une énorme tête d'éléphant, aussi blanche que les ventilateurs, rejoint le groupe. Le spectateur peut l'interpréter comme une créature hybride ou bien comme une apparition du dieu Ganapati ${ }^{15}$. Le groupe en oublie les ventilateurs pour s'étonner de cette présence à la fois incongrue et divine. Finalement, ni les personnages ni les spectateurs ne savent plus si ces objets sont un cadeau du ciel ou de la modernité. C'est pourquoi, à chaque fois que l'élément air surgit dans une scène de Bamboo Blues, il questionne ces transitions où circulent les signes ainsi que le jeu de liberté d'interprétation et ses limites quand on passe sans cesse d'une culture à l'autre.

\section{De l'air entre les cultures}

Lorsque deux danseurs éventent un couple d'amoureux avec des morceaux de carton, ils rappellent le manque d'espace privé dans la vie indienne. Dans une autre scène, l'image d'une statue de temple bariolée représentant un gandharva ${ }^{16}$, est démultipliée en six : trois fois sur le rideau de fond et trois fois sur le sol ${ }^{17}$. Dans ce décor, sept filles et sept garçons face-àface viennent jouer bruyamment en se lançant des châles, les rattrapant, s'accrochant ou s'enroulant avec. L'élément air est leur milieu commun, la divinité projetée semble voler autant que les tissus réels, créant un sentiment de joie et de tolérance dans les échanges, qu'ils aient lieu dans les mondes invisible ou visible, céleste ou terrestre. Tous ces mondes coexistent avec l'air comme seule frontière, milieu privilégié dans lequel les contraires peuvent coexister, ce qui est aussi un principe fondamental de la société indienne ${ }^{18}$. Dans ce sens, à chaque fois que les danseurs disparaissent dans le noir, les spectateurs cherchent à vivre cette transition comme un espace de pensée, pour questionner la signification culturelle d'un geste, d'une image ou d'une action, ou se heurter à une incompréhension totale des $\operatorname{codes}^{19}$. Mais l'enchaînement effréné des saynètes ne lui en laisse guère le temps : le plaisir du

\footnotetext{
${ }^{15}$ Ganapati : dieu le plus populaire du panthéon hindou, il est représenté par un corps d'homme avec une tête d'éléphant. Dans les représentations de Bamboo Blues à Calcutta et à Delhi notamment, c'est une femme qui porte cette tête et l'ôte sur la scène.

${ }^{16}$ Les Gandharva sont, dans la mythologie hindoue, les messagers entre les dieux et les humains. Ces musiciens célestes sont associés aux Apsaras, les danseuses célestes.

${ }^{17}$ Ces ressemblances entre les corps des divinités projetées et les corps réels - dans leur gestuelle et dans leur habillement -, met en valeur ce trait idéologique commun aux Indiens : la supériorité du collectif sur l'individu, de l'impersonnel sur le personnel.

${ }^{18}$ Cf. Christophe Jaffrelot, L'Inde contemporaine, éd. Fayard, 2006, Introduction, pp. 11-21.

${ }^{19}$ Anne Linsel, Pina Bausch et le Tanztheater en tournée, op. Cit.: pendant la tournée de 1994 du Tanztheater en Inde, la réalisatrice du film consacré à ce voyage fait la remarque suivante : « la compagnie a assisté à un cours de danse donné à des enfants de bidonvilles. Les jeux sont aussi présents sur scène, car Pina Bausch et ses
} 
rythme et de ses vitesses l'emporte.

Cette fonction esthétique de l'air à la fois comme ambiance de nature et comme milieu rythmique de relation entre les êtres rend caduque l'idée d'un théâtre comme imitation d'une réalité extérieure ou représentation d'un monde déjà donné ${ }^{20}$. La Danse-théâtre de Pina Bausch capte des forces et joue rythmiquement avec l'action de ces forces sur le corps, avant même que ce dernier soit reconnu dans une forme particulière et culturellement établie. Il s'agit au fond d'opposer le figuratif (la transformation des formes) au figural (la déformation des corps). Gilles Deleuze le formule ainsi : « la forme rapportée à la sensation (Figure), c'est le contraire de la forme rapportée à un objet qu'elle est censée représenter (...). La Figure est une Idée, c'est-à-dire une image qui donne à penser $^{21}$ ». La Figure ne renvoie donc pas au personnage joué par le danseur mais aux jeux rythmiques l'isolant, le déformant, l'effaçant selon les rapports de forces créés entre lui et les matériaux de son art. La danse s'inscrit dans cette alternance infinie entre les corps qui s'approchent, s'enlacent et se repoussent. Ce battement à trois temps devient visible et sensible au point de faire oublier aux spectateurs les individus en jeu. L'impersonnel est cet état d'être, tel un frisson qui tantôt dissipe le corps vers quelque chose d'inconnu, d'imprévisible, de virtuel et tantôt le comprime ici et maintenant, dans une action précise et réelle, parfois jusqu'à l'épuisement de l'artiste sur scène. Dans l'une des scènes, un danseur, assis sur le sol, déplie et plie sans cesse une jambe, de manière très mécanique, poussant ou tirant son genou, en augmentant progressivement sa vitesse jusqu'à ce qu'il soit physiquement impossible de continuer. Ce mouvement est un agencement de sensations jusqu'à devenir une vibration, oscillant sans cesse entre la diastole (la disparition dans la Figure) et la systole (la concentration dans un geste actuel ${ }^{22}$ ). L'air joue un double jeu : soit les corps s'oxygènent et se dynamisent en se repoussant, préservant ainsi assez d'espace rythmique entre eux et entre leurs mouvements, soit ils s'étouffent dans leurs enlacements ou s'asphyxient dans la répétition effrénée d'un même geste. Lorsqu'il n'y a plus d'intervalles rythmiques ou physiques, le geste ou le mouvement s'épuisent.

\section{L'air déforme}

La fonction ambivalente de l'air dans Bamboo Blues se présente de diverses manières, notamment dans une série de déformations animales du corps au tout début du spectacle. Après le solo d'ouverture d'une danseuse en rose, quatre danseuses vêtues de longues robes de soirée occidentales, chacune d'une couleur éclatante et unie - rose foncé, verte, bleu et rouge - entrent successivement en marchant lentement. On les voit mâcher de manière exagérée. Venant s'asseoir à l'avant-scène, face au public, elles prennent des poses nonchalantes. Le regard fixe, inexpressif et perdu au loin, les voilà devenues des vaches en train de ruminer. Elles invitent le public dans un espace bien particulier, non pas une prairie occidentale, mais une rue indienne où ce genre de face-à-face est quotidien puisque les vaches s'y déplacent

\footnotetext{
danseurs remontent souvent à l'enfance pour y trouver l'une des sources de nos perturbations, de notre violence et de notre incapacité à aimer. Ce regard sur soi-même et sur son histoire personnelle est totalement étranger aux Indiens et à leur art de la danse ».

${ }^{20}$ Même lorsque la référence au code traditionnel indien est explicite, elle ouvre à une liberté d'interprétation. Prenons l'exemple de la scène où une femme en rouge entre par le rideau de fond et vient jusqu'au public en répétant toujours le même geste d'une main, partant de la bouche et dépliant les doigts tout en soufflant légèrement. Le spectateur occidental a l'impression qu'un baiser lui est envoyé, le connaisseur des mudrâ, le langage des mains propre aux acteurs-danseurs, comprend qu'il s'agit d'une parole qui nous est soufflée.

${ }^{21}$ Gilles Deleuze, Deux régimes de fous. Textes et entretiens 1975-1995, éd. Lapoujade, 2003, p. 194.

${ }^{22}$ Gilles Deleuze, Francis Bacon. Logique de la Sensation, éd. de La Différence, Paris, 1981, p. 65.
} 
librement. Sans doute, ce sujet d'étonnement pour un touriste arrivant brutalement là-bas est-il devenu un cliché. Des animaux impassibles, perturbant souvent la circulation, imperturbables malgré le bruit, spectateurs dont le regard ne s'attache à aucun objet. Voici tout-à-coup le public de Bamboo Blues pris dans ces blocs de sensations pleines de couleurs, de sons et de forces et dans ce lieu de spectacle vivant qu'est la ville indienne du point de vue des bovidés. Un malaise émerge des gradins, interrogeant une certaine insensibilité du regard, jusqu'à son côté inhumain : en quoi ces séduisantes jeunes femmes deviennent-elles des animaux et en quoi cela nous concerne-t-il quand nous avons à affronter leur regard imperturbable et inexpressif

La scène suivante étonne de la même manière : un homme entre avec un tabouret et s'assoit. Deux des femmes-vaches viennent s'accroupir auprès de lui. Chacune allume un briquet sous ses plantes de pieds nus. Tous les trois sourient, l'homme reste stoïque, sans réagir à la brûlure possible. Pendant ce temps, la troisième de ces femmes bovidiennes se déplace au fond de la scène où l'on vient de placer une table. Elle s'y installe à plat ventre tout en nous regardant, le sourire aux lèvres. D'une contorsion acrobatique et savante, elle pose ses pieds sur ses épaules et sur son crâne ${ }^{23}$. Dans la scène suivante, les danseurs entrent deux par deux et chaque couple plie en quatre un tissu blanc de plusieurs mètres de long. Soudain, au même moment, tous les draps sont soulevés vers le haut par un courant d'air et se gonflent comme des voiles entre les mains de chaque couple. L'élément air rythme donc les mouvements sans maîtrise possible de l'être humain. Il va jusqu'à inverser le geste quotidien qui habituellement plie un drap en le laissant retomber vers le sol de tout son poids. Tout est souple dans ces scènes, se plie, se déplie, se déforme à souhait, même le corps humain. A chacune de ces actions, les danseurs regardent les spectateurs et leur sourient, un peu comme après un numéro de variété où une prouesse vient d'être accomplie ou peut-être pour marquer un certain recul ironique vis-à-vis des personnages qu'ils interprètent ${ }^{24}$. Dans tous ces exemples où le spectateur est pris à témoin par le regard ou le sourire insistant des danseurs, un interstice invisible se crée entre les deux espaces de la scène et de la salle. Il ouvre un court moment de réflexion sur l'interculturalité, autant dans la distanciation de l'artiste d'avec son jeu que dans la pensée du spectateur lorsqu'il arrête d'émettre un jugement, un cliché ou un présupposé sur des scènes apparemment absurdes et sans logique narrative.

\section{Variations rythmiques des rideaux de scène}

La scène des vaches en train de ruminer fait retour juste avant l'entracte. La séquence qui la précédait en ouvrant le spectacle, le solo de la danseuse en rose, est reconduite également. Les ruminantes ne sont plus quatre mais six, regroupées tout près du premier rang des spectateurs : une orange, une rose, une rouge, une blanche parsemée de grosses fleurs, une violette et une verte. Un homme entre et dit : "c'est l'entracte ». Les femmes bovidiennes ressortent. Néanmoins, il est devenu impossible de parler d'une répétition de la scène initiale dans la mesure où un élément scénique a été ajouté pendant le solo et la pose des ruminantes. En effet, placé au bord de la coulisse à gauche du public, un homme se met à tirer un rideau géant jusqu'au centre de la scène sur lequel est projetée une image : une enfilade de cocotiers au bord d'une route. A plusieurs reprises, il replie le rideau, l'image disparaît puis ce même homme le déplie et la succession d'arbres réapparaît. Pendant ce temps, la danseuse en rose

\footnotetext{
${ }^{23}$ Il s'agit de la posture de l'arc en yoga, appelée Dhanurâsana, décrite dans l'ouvrage suivant : B.K.S Iyengar, Yoga Dipika, éd. Buchet/Chastel, Paris 1978, p. 296.

${ }^{24}$ Leonetta Bentivoglio / Francesco Carbone, Pina Bausch vous appelle, éd. L'Arche, Paris, 2007, p. 32.
} 
continue son solo. On la voit tantôt en transparence derrière l'écran, tantôt devant lui.

Ce rideau blanc agit en tant qu'espace interstitiel, créant quatre rythmes différents. Ils sont presque simultanés et désorientent le spectateur en lui donnant à voir plusieurs scènes en même temps. Au premier niveau, la musique est coordonnée avec les mouvements de la danseuse en rose, installant le sentiment d'un ordre du mouvement et d'une mesure régulière. En second lieu, les dépliements/repliements successifs du rideau donnent l'illusion au spectateur de se déplacer en hauteur - dans un bus indien - puisqu'on ne voit que la partie supérieure des arbres qui défilent de gauche à droite, signifiant que nous nous déplaçons dans le sens inverse des aiguilles d'une montre. A un troisième niveau, la présence des ruminantes immobiles se donne comme un point fixe regardant les spectateurs en voyage. Enfin, la transparence du rideau révélée par la présence de la danseuse qui virevolte à contresens des cocotiers, rend la scène du voyage illusoire. Mais en même temps, elle déréalise cette danseuse, qui n'apparaît plus que comme une tache rose aérienne en mouvement derrière les arbres. Dans la dernière grande scène de Bamboo Blues, cette séquence est répétée avec des nuances : la danseuse en rose se met à crier puis appelle un homme avec qui elle roule à terre. Des danseurs sont présents et observent la scène : un couple sur un lit roulant - déjà là dans la première partie du spectacle - regarde le duo et rit. L'écran sur lequel défilent les images des arbres finit par jouer le rôle d'un vrai rideau de scène, mais à l'inverse de ce qui se fait habituellement. Au lieu d'être déplié sur toute la longueur du plateau, il est replié vers les coulisses. Les danseurs entrent en se donnant la main et viennent saluer le public.

Il existe dans Bamboo Blues un second rideau qui, lui, est permanent, placé tout au fond de la scène. Fait de multiples plis et pans découpés, il se balance sous l'effet d'une brise artificielle, révélant ou voilant de minuscules espaces par lesquels entrent et sortent les danseurs, parfois en virevoltant. Les mouvements s'accomplissent toujours avec lenteur, laissant donc le temps au spectateur de contempler ces interstices. Ils détournent ainsi son attention des autres danseurs qui apparaissent ou disparaissent à toute vitesse sur les côtés, dans l'obscurité des coulisses.

Avant d'être un élément particulier de la scénographie, cet immense tissu blanc de Bamboo Blues s'agence d'abord comme un bloc de sensation : fraîcheur-air-légèretéouverture-plaisir. Parce qu'il apparaît au tout début de la première scène doucement agité et balancé par une brise, il ne représente ni limite, ni frontière ni décor : il devient l'air luimême, sa matière visible, la sensation aérienne qu'il nous donne pendant tout ce spectacle. Les corps des danseuses s'y frottent pendant leurs solos, leurs bras ou leur chevelure y disparaissent quelques courts instants. Effaçant les frontières entre les éléments, l'air qui semble se «matérialiser » en tissu blanc contamine même la terre : la projection de l'image géante d'une forme blanche et mobile sur le rideau envahit également le plateau. Le spectacle débute dans le silence avec l'entrée d'une danseuse en robe longue, une petite tache rose vif perdue dans l'immensité des soixante dix-huit pans de tissus blancs, tombant à l'aplomb d'une quarantaine de tringles suspendues le plus haut possible en fond de scène. Si l'immobilité était de mise, le spectateur pourrait y voir un conventionnel rideau de théâtre plissé. Mais dans le moment silencieux du commencement, une danse se devine dans la multitude d'interstices entre ces tissus balancés doucement par une brise venue de nulle part. Des images surgissent pour ceux qui ont déjà voyagé en Inde puis disparaissent aussitôt : le linge qui sèche n'importe où dehors, constitué principalement de ces immenses pièces de plusieurs mètres de long, appelés dhotis pour les hommes et saris pour les femmes. Le spectateur comprend très vite que ce fond de scène est le lieu de l'air : plusieurs solos de femmes en rose ou en violet $y$ évoluent, au point que leurs mouvements, comme ceux des rideaux, semblent causés ou 
débordés par ce petit vent. Il y joue avec leurs longues chevelures noires étirées entre les doigts ou renversées en avant, balancées au rythme de ces grands panneaux mobiles et légers. Certains mouvements surgissent littéralement comme des effets de l'élément air, telle l'apparition de cette femme en rouge qui sort lentement de ces coulisses blanches, traverse la scène en oblique, ne cesse de souffler doucement sur sa main et vers le public, puis repart dans le noir - un homme lui court après, en vain.

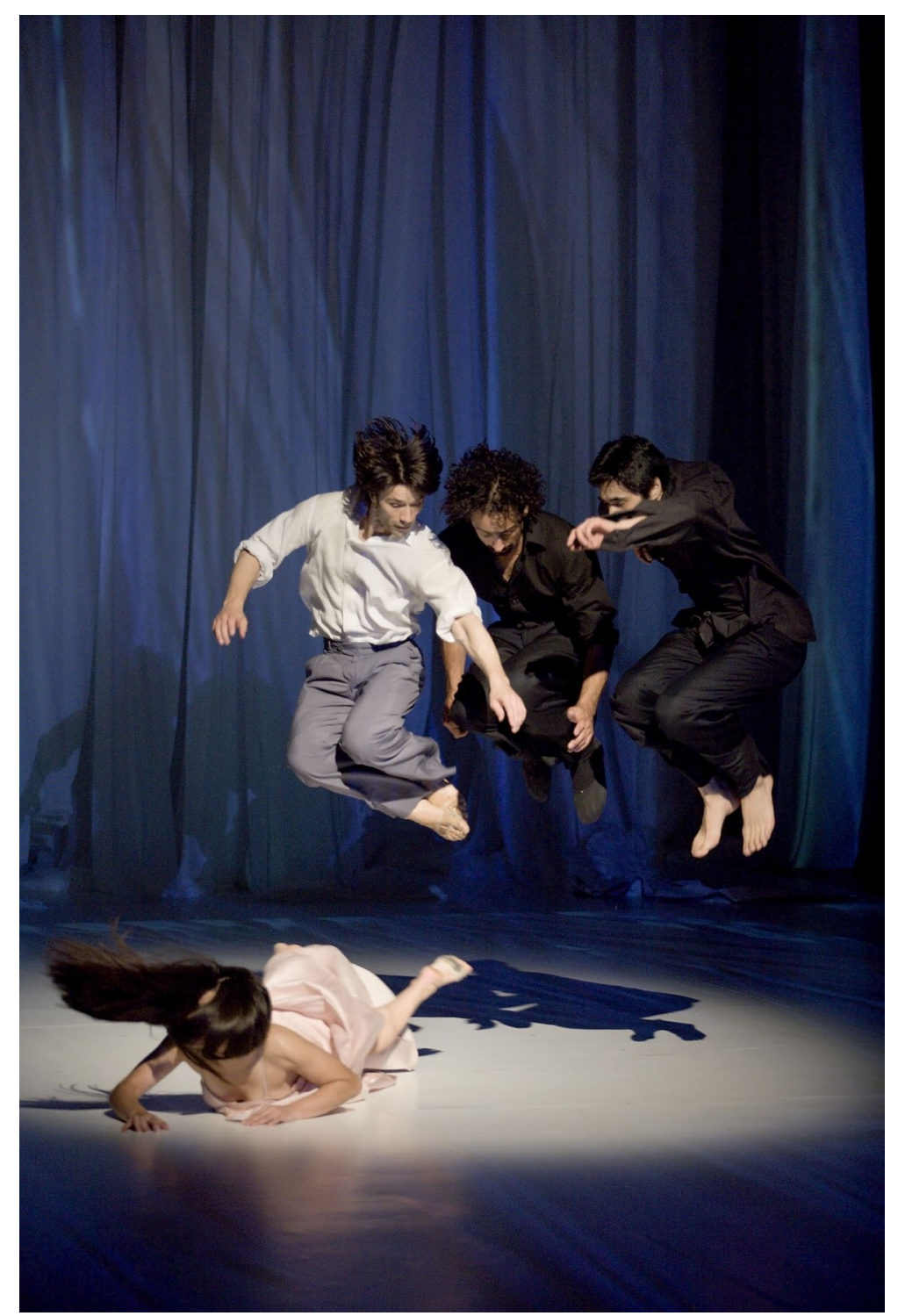

Bamboo Blues, Pina Bausch - (C) Théâtre de la Ville, Paris

Bamboo Blues donne ainsi à voir deux types de transition entre les scènes. La première fabrique des interstices rythmiques pratiquement invisibles pour les spectateurs, avec toutes sortes de déplacements circulaires des danseurs, donnant une impression de continuité non narrative. La seconde, créée par l'élément air, se fonde à la fois sur des intervalles visibles sur le plateau et sur des espaces libres de réflexion entre les cultures qui mettent en jeu les significations des gestes et des actions.

Nous venons de comprendre, avec ces jeux de rideaux, comment derrière chaque mouvement effectuant un déplacement réel dans l'espace, des artifices dramatiques ou 
scénographiques créent d'infimes intervalles temporels annulant toute perception d'ordre chronologique. Lorsque dans l'une des scènes, des myriades de taches de lumière se déplacent de manière aléatoire sur le rideau de Bamboo Blues, il ne s'agit pas d'une transition entre deux événements successifs (un couple qui danse avec une rose rouge, puis la projection du film sur le $c h h a u^{25}$ ) mais le rappel, par une impression visuelle forte, que toutes les translations sont habitées par une pulsation rayonnante et rythmique. Lorsque la danseuse évolue en transparence derrière le rideau diaphane, le spectateur cesse de s'attacher au signe représenté sur l'écran (le jeu du soleil dans les arbres ou dans les rideaux ${ }^{26}$ ) ou sur le plateau (la danseuse). Il passe de « l'image-mouvement à l'image-temps ${ }^{27}$ », de la translation de formes à la vibration, autrement dit à la transformation intensive de variations d'affects et de forces. Par conséquent, à cet instant, le spectateur cesse d'agir ou de réagir avec des constructions de clichés et d'histoires. Il oublie de relier ce qu'il voit à une narration possible. Il rompt avec la croyance que l'espace est un lieu vide à remplir par des événements visibles. Il devient simplement présent à cette multiplicité de fractures dynamiques.

Katia Légeret

Maître de conférences, Université Paris 8

katia.legeret@univ-paris8.fr

\section{Références de l’article :}

Katia Légeret, «Rythmes, air et tissus blancs : l'art des transitions entre les cultures dans Bamboo Blues de Pina Bausch», Agôn [En ligne], №1 : Interstices, entractes et transitions, L'interstice en scène, Dossiers, mis à jour le : 09/12/2008, URL : http://agon.enslsh.fr/index.php?id=719.

\footnotetext{
${ }^{25}$ Le chhau est un style traditionnel de danses dramatiques masquées de l'est de l'Inde, marquées par les arts martiaux.

${ }^{26}$ Dans Agua (2001), il y a également une projection sur les murs et le sol de palmiers se balançant dans le vent, suivi d'un film sur les percussionnistes brésiliens et dans Masurca Fogo (1998), la seconde partie reprend les thèmes du début en ajoutant des projections de photos de voyage, alternant des voyageurs en bus regardant le paysage avec la présence d'oiseaux rares.

${ }^{27}$ Gilles Deleuze, Cinéma 2 - L'image temps, coll. "Critique", éd De Minuit, Paris, 1985, pp. 18-20.
} 